\title{
ROLE OF STRAIN CRYSTALLIZATION IN THE FATIGUE RESISTANCE OF DOUBLE NETWORK ELASTOMERS
}

\author{
P. G. SANTANGelo, C. M. Roland* \\ NaVAl Research Laboratory, Chemistry Division \\ CODE 6120, WASHINGTON D.C. 20375-5342
}

\begin{abstract}
Double networks were prepared from guayule rubber (GR), deproteinized natural rubber (DPNR), and styrenebutadiene rubber (SBR), and their properties compared to conventional "single networks" having the same crosslink density. Substantial residual strains (> 150\%) were obtained in all double networks, whereby the modulus parallel to the residual strain was enhanced. For the two strain-crystallizing elastomers, the fatigue resistance of the double networks (for extensions parallel to the residual strain) was higher than for their single network counterparts. Moreover, the guayule rubber, which is more strain-crystallizable than DPNR, exhibited the greater enhancement. For the amorphous SBR, on the other hand, the network structure had an insignificant effect on the fatigue life. These results demonstrate that longer mechanical fatigue lifetimes in double network rubbers are a consequence of their intrinsic orientation. This provides the capacity to retain crystallinity at the front of growing cracks, even in the absence of stress. The origin of the improved fatigue resistance is similar to the mechanism responsible for the better performance of strain-crystallizing rubbers subjected to non-relaxing cyclic deformations.
\end{abstract}

\section{INTRODUCTION}

A double network elastomer refers to rubber crosslinked twice, the second time while in a deformed state. While double networks can be formed inadvertently due to chain $\operatorname{scission}^{1,2}$ or strain-induced crystallization, ${ }^{3-5}$ the potential for improved mechanical properties in such materials has elicited much interest. The modulus of NR double networks increases with residual strain, after an initial minimum. ${ }^{6,7}$ Thus, when appropriately prepared, double networks can have higher modulus than single networks of the same modulus. Previous work ${ }^{8,9}$ also demonstrated that natural rubber double networks have better fatigue properties, an effect we tentatively ascribed to strain-induced crystallization. The presence of a double network has been shown to reduce the deformation necessary to induce crystallization of natural rubber. ${ }^{10}$

This work compares the fatigue life of strain crystallizing rubbers to that of non-crystallizing elastomers. Only the response of double networks to deformations parallel to their residual strain is investigated. Although double network technology has been successfully implemented with filled rubber, ${ }^{11-13}$ our focus herein is on gums.

\section{EXPERIMENTAL}

Networks were made from three polymers, a deproteinized Hevea rubber (DPNR, from H.A. Astlett Co.), an ASTM D 2227 grade 5 guayule rubber (GR, provided by S.F. Thames of the University of Southern Mississippi via the U.S. Department of Agriculture's Cooperative State, Research, Education and Extension Service), and a styrene-butadiene (SBR) copolymer (1502NN from Goodyear). To the DPNR, GR, and SBR, 2.8, 2.9, and $0.25 \mathrm{phr}$ respectively of dicumyl peroxide (Varox DCP-R, RT Vanderbilt Co., Inc.) were incorporated on a two-roll mill. These peroxide levels were empirically determined to yield the same low-strain modulus in the double networks. Peroxides are preferred for crosslinking of double networks, since sulfur vulcanizates are susceptible to reversion during the second curing, which is done in a strained state. Isotropic sheets were compression molded for $70 \mathrm{~min}$ at $135^{\circ} \mathrm{C}$ to obtain the initial network. Strips were cut from these cured sheets, and stretched to $\sim 280 \%$ ( $230 \%$ for the SBR), as deter-

\footnotetext{
* Corresponding author. Ph: 202-767-1719; Fax: 202-767-0594; email: roland@nrl.mil
} 
mined by fiducial marks. While stretched, the samples were further cured by heating in a vacuum oven for $20 \mathrm{~min}$ at $115^{\circ} \mathrm{C}$, followed by $10 \mathrm{~min}$ at $160{ }^{\circ} \mathrm{C}$. Additional unstrained samples were also subjected to the second curing, simultaneously with the stretched samples. We refer to these as "single networks," since there is no distinction between their first and second sets of crosslinks. These single networks should have the same crosslink density as the double network, assuming that the chemical reactivity is independent of strain, as expected for rubbery polymers. All testing was done at room temperature and, for the double networks, parallel to the extension during the second curing. The stress/strain measurements were carried out using an Instron 4206 and optical extensometer (H.W. Wallace Co.), at an extension rate of $2.0 \%$ per second. Recoverable strain energies were calculated from the retraction curve for the third cycle. Tensile fatigue testing employed a Monsanto Fatigue to Failure Tester (ASTM D4482-85) interfaced to a PC. A minimum of five specimens was tested at various strains ranging from $61 \%$ to $136 \%$ elongation. The median values of the number of cycles to failure (i.e., the fatigue life) are reported herein.

\section{RESULTS AND DISCUSSION}

The equilibrium residual strain (permanent set) for each of the six elastomers measured subsequent to the second curing stage is listed in Table I. Also included in the table is the equilibrium modulus at $10 \%$ elongation. By design, the double networks all have the same low-strain modulus, to within the experimental error. Although the single networks have the same crosslink density as the double networks, in every case their modulus is lower than that of the corresponding double network. This is a consequence of the high residual strains obtained for the latter. ${ }^{6,7}$

TABLE I

Mechanical Response of Networks

\begin{tabular}{cccc}
\hline Rubber & Network & $\begin{array}{c}\text { Residual } \\
\text { Strain }\end{array}$ & $\begin{array}{c}\text { Modulus* } \\
(\mathrm{MPa})\end{array}$ \\
\hline SBR & Single & 0 & 0.66 \\
& Double & $155 \%$ & 1.05 \\
\hline DPNR & Single & 0 & 0.81 \\
& Double & $240 \%$ & 1.03 \\
\hline GR & Single & 0 & 1.05 \\
& Double & $190 \%$ & 1.11 \\
\hline
\end{tabular}

* Equilibrium stress divided by strain $(=10 \%)$.

In Figure 1 are shown the stress-strain curves for the six networks. Obtained during slow retraction, these represent near equilibrium data. ${ }^{14,15}$ Notwithstanding the approximate equivalency of their low-strain moduli, the mechanical responses of the networks are quite different at higher extensions. For every case, the stresses for the double network greatly exceed that for the single network. Since the crosslink densities are equal, this elevation of the high-strain modulus, even beyond that seen at low strains (Table I), represents one of the advantages of utilizing a double network. 


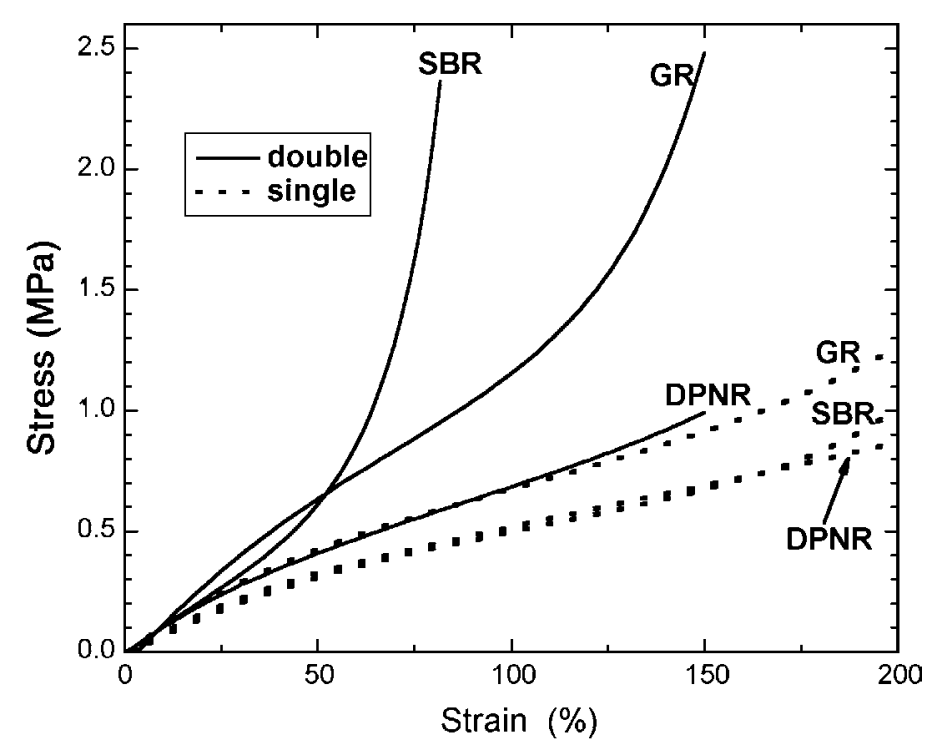

FIG. 1. - Tensile stress measured for the six networks as a function of elongation (parallel to the residual strain for the double networks). For the single networks, the data were obtained during retraction from $200 \%$. This maximum strain was reduced for the double networks, because their elongation to break was too short.

It was also observed that the double networks were more brittle (reduced elongation) than single networks. This reflects finite extensibility of the chains of the initially-formed network. These network chains are stretched during the second cure step, and are thus extended in the stress-free reference state. This gives rise to the residual strain of the double network, and likely contributes to the elevated stresses at large strains. The data in Figure 1 also appear to suggest that the tensile strength of the double networks is enhanced; however, this is not the case. Measurements on the single networks were arbitrarily limited to strains not exceeding 200\%. An earlier study, based on data obtained for several hundred samples, demonstrated that at constant crosslink density, the tensile strength of double networks is lower than for single networks. ${ }^{6}$

Our main interest is the relative fatigue lifetimes of the two network types. Over a wide range of strains, the rate of crack propagation varies as a power law in the tearing energy (strain energy release rate). ${ }^{16,17}$ Since this tearing energy is essentially proportional to the recoverable strain energy, W, the fatigue life (integral of the crack propagation rate) can be expressed as

$$
N=a W^{b}
$$

in which $\mathrm{a}$ and $\mathrm{b}$ are material parameters. Thus, a double logarithmic plot of fatigue life versus strain energy should be linear.

Figure 2 shows the results for the DPNR networks. Over most of the range, the double networks exhibit a longer fatigue life. This agrees with results of our earlier study on natural rubber double networks. ${ }^{6}$ As also observed in the earlier work, ${ }^{6}$ the scatter in the data is somewhat higher than for the single networks. Nevertheless, except at the highest strain energies, $\mathrm{N}$ is significantly larger for the DPNR double networks. 


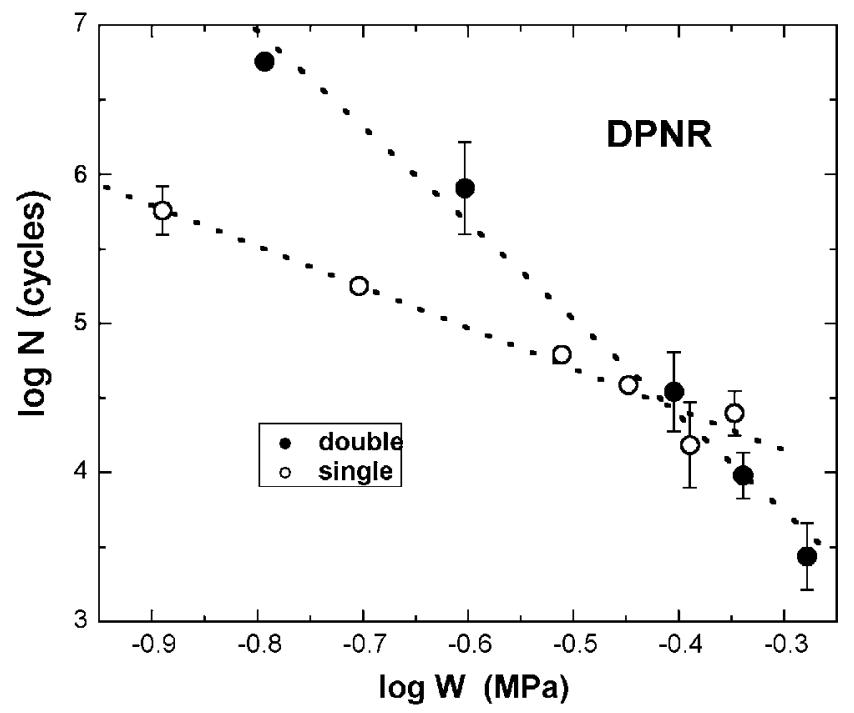

FIG. 2. - The logarithm of the mechanical fatigue life as a function of the logarithm of the recoverable strain energy for single (hollow symbols) and double (solid symbols) networks of deproteinized natural rubber. The latter were measured parallel to the residual strain. The standard deviation in the measured fatigue life is as shown, except for those points for which the scatter is smaller than the symbol size. The dotted lines represent the best fits to Equation 1.

The parameters obtained from the fit of the data to Equation 1 are listed in Table II. Generally, a quadratic dependence of $\mathrm{N}$ on strain energy release rate is found for natural rubber. ${ }^{18}$ While for DPNR single networks, the exponent in Equation 1 is close to -2 , for the double networks $\mathrm{b} \approx-6$. This stronger dependence of $\mathrm{N}$ on strain energy for double networks was noted previously, ${ }^{9}$ although its origin remains unclear.

TABLE II

FATIGUE PROPERTIES OF NETWORKS

\begin{tabular}{ccccc}
\hline Rubber & Network & $\mathrm{a}^{*}$ & $\mathrm{~b}^{*}$ & $\begin{array}{c}\mathrm{N}^{\dagger} \\
\text { (kcycles) }\end{array}$ \\
\hline \multirow{2}{*}{ SBR } & Single & $1.52 \pm 0.56$ & $-4.53 \pm 1.19$ & 17 \\
& Double & $2.59 \pm 0.17$ & $-3.26 \pm 0.38$ & 35 \\
\hline DPNR & Single & $3.33 \pm 0.16$ & $-2.74 \pm 0.27$ & 93 \\
& Double & $1.89 \pm 0.24$ & $-6.44 \pm 0.46$ & 560 \\
\hline GR & Single & $4.14 \pm 0.05$ & $-1.72 \pm 0.11$ & 150 \\
& Double & $3.57 \pm 0.16$ & $-6.23 \pm 0.66$ & 2,000 \\
\hline
\end{tabular}

${ }^{*}$ Equation 1 (units $\left.\equiv \mathrm{MPa}\right) .{ }^{\dagger} \mathrm{W}=0.25 \mathrm{MPa}$.

In Figure 3 are displayed fatigue lifetimes for the GR networks. Qualitatively, these data are similar to the results for DPNR in Figure 2. The GR double networks exhibit substantially better fatigue resistance than the single networks, with the differences diminishing at larger W. The cut growth parameters (Equation 1) are listed in Table II. Again, we find for the single network the exponent is approximately -2 , whereas for the GR double networks $b \approx-6$. 


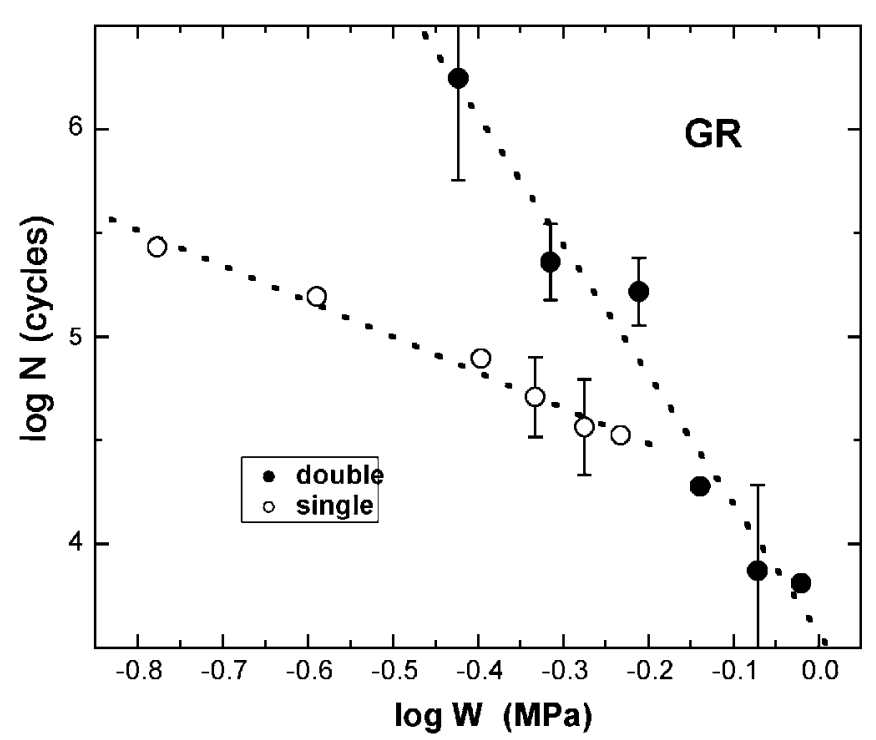

FIG. 3. - The logarithm of the mechanical fatigue life as a function of the logarithm of the recoverable strain energy for single and double networks of guayule rubber. The latter were measured parallel to the residual strain. The symbols are the same as in Figure 2.

It has been shown previously that when compared at equal modulus, single networks of GR have better failure properties than DPNR. ${ }^{19,20}$ This is due to enhanced crystallizability; specifically, strain-induced crystallization is induced at lower strains in GR than in DPNR. ${ }^{21}$ Accordingly, if the superior fatigue properties of double networks are a consequence of strain crystallization, the effect should be more prominent in GR. This is indeed the case. Over the range of strain energies where the respective data overlap, $\mathrm{N}$ for the GR double networks exceeds that for the double networks of DPNR.

To quantify this, we calculate from the cut growth parameters the fatigue life for a strain energy equal to 0.25 ( $\log \mathrm{W}=-0.6$ ). For both network types, the fatigue life for GR exceeds $\mathrm{N}$ for DPNR (Table II). Moreover, the enhancement resulting from a double network in comparison to the corresponding single network is much larger for the GR; that is, better advantage is taken of double networks by using a more readily strain-crystallizing elastomer.

Figure 4 displays fatigue life data for the SBR networks. The behavior of this non-crystallizing elastomer is distinct from the results for the GR and DPNR in several respects. For both network types, SBR has poorer fatigue resistance than the other rubbers. For example, $\mathrm{N}$ at $\mathrm{W}=$ $0.25 \mathrm{MPa}$ for the SBR double network is smaller by a factor of 3 than the worst performing network of the other two materials (Table II). The difference between the single and double SBR networks is also quite small. In fact, within the experimental error, which was relatively large for the SBR, any difference between the two networks is barely significant. 


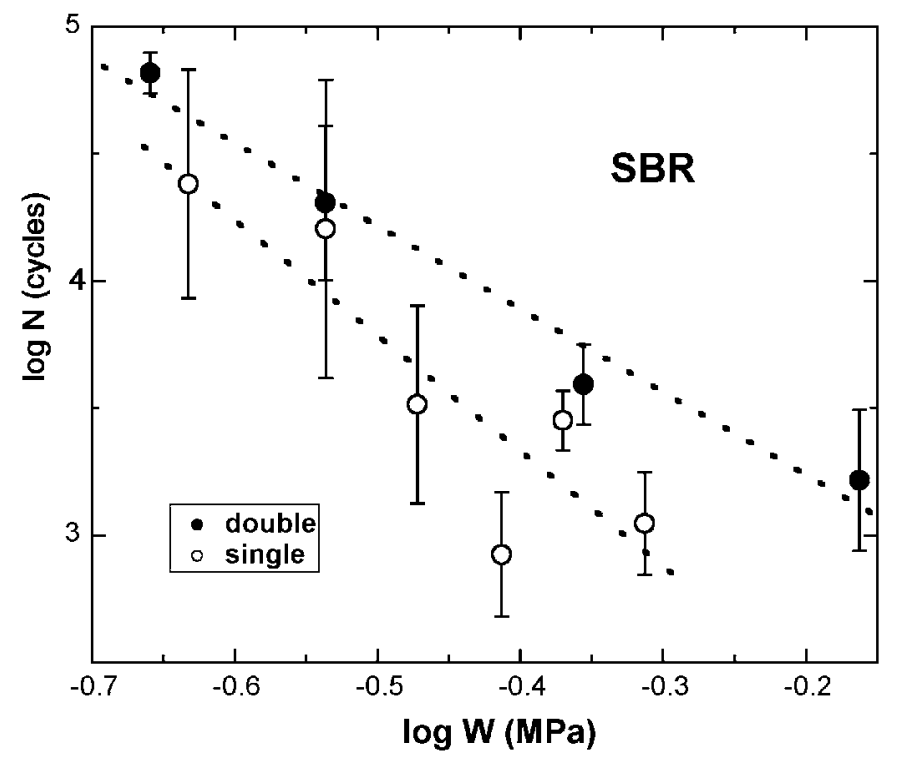

FIG. 4. - The logarithm of the mechanical fatigue life as a function of logarithm of the strain energy for single and double networks of the SBR. The latter were measured parallel to the residual strain. The symbols are the same as in Figure 2.

Note also in Table II that only for the SBR is the prefactor in Equation 1 larger for the double network than for the single network. This reinforces the point that enhancement of fatigue properties arises due to strain crystallization. For the SBR, there is only a modest difference between the respective slopes in Figure 4 for the single and double networks. The strain energy dependence of the fatigue life is stronger than quadratic, $b \approx-4$, as generally found. ${ }^{16,22}$

\section{CONCLUSIONS}

The principal purpose of this study was to determine if improvements in mechanical fatigue properties, observed previously for double networks of natural rubber, ${ }^{8}$ are a consequence of strain crystallization. The presence of a double network itself is known to enhance strain crystallizability. ${ }^{10}$ All double networks were prepared to have large residual strains $(>150 \%)$. To achieve this, a substantial fraction of the crosslinks was apportioned to the second network, with a large strain imposed during its formation. ${ }^{6}$ As a result, modulus enhancement was observed for the three rubbers studied herein (Table I).

The double networks based on cis-1,4-polyisoprene, a strain-crystallizing polymer, exhibited enhanced fatigue performance in comparison to single networks having the same crosslink density (Table II). Moreover, this enhancement was greater (by more than a factor of two at $\mathrm{W}=$ $0.25 \mathrm{MPa}$ ) for the GR, which is more readily strain-crystallized than is DPNR. ${ }^{21}$ The superiority of GR double networks was obtained despite residual strains that were lower than for the DPNR (Table I). On the other hand, double networks of SBR, a non-crystallizing rubber, showed negligible differences in fatigue resistance from SBR single networks. Thus, the hypothesis that strain crystallization underlies the superiority of double networks is affirmed.

In the fatigue experiments, the stress returned to zero for each deformation cycle ("relaxing conditions"). Under non-relaxing conditions, the fatigue life increases markedly for strain-crystallizing rubbers. ${ }^{16,23}$ By a similar mechanism, annealing in a strained state can increase the fatigue resistance of such rubbers. ${ }^{24} \mathrm{We}$ believe that the better fatigue resistance of strain-crystallizing double networks is due to their ability to retain orientation when the stress goes to zero 
at the minimum of the strain cycle. This intrinsic orientation is seen in the birefringence of double networks in an relaxed (zero stress) state. ${ }^{10,25}$ This orientation stabilizes any crystallinity at the front of growing cracks, thereby inhibiting their propagation. Thus, the origin of the enhanced fatigue life for double networks of strain-crystallizing rubbers is analogous to the mechanism giving rise to better fatigue resistance under non-relaxing deformations of single networks of such materials.

\section{ACKNOWLEDGEMENT}

This work was supported by the Office of Naval Research.

\section{REFERENCES}

${ }^{1}$ A. V. Tobolsky, Y. Takahashi, and S. Naganuma, Polym. J. 3, 60 (1972).

${ }^{2}$ K. T. Gillen, Macromolecules 21, 442 (1988).

${ }^{3}$ P. J. Flory J. Amer. Chem. Soc. 78, 5222 (1956).

${ }^{4}$ L. Mandelkern, D. E. Roberts, A. F. Diorio and A. S. Posner, J. Amer. Chem. Soc. 81, 4148 (1959).

${ }^{5}$ R. A. M. Hikmet, J. Lub, and P. M. Vanderbrink, Macromolecules 25, 4194 (1992).

${ }^{6}$ P. G. Santangelo and C. M. Roland, Rubber Chem. TeChnol. 67, 359 (1994).

${ }^{7}$ S. Kaang, D. Gong, and C. Nah, J. Appl. Polym. Sci. 65, 917 (1997).

${ }^{8}$ P. G. Santangelo and C. M. Roland, RubBer Chem. TeChNOL. 68, 124 (1995).

${ }^{9}$ S. Kaang and C. Nah, Polymer 39, 2209 (1998).

${ }^{10}$ C. M. Roland and M. L. Warzel, Rubber Chem. Technol. 63, 285 (1990).

${ }^{11}$ C. M. Roland and K. L. Peng, Rubber Chem. Technol. 64, 790 (1991).

${ }^{12}$ W. F. Reichert, D. Goritz and E. J. Duschl, Polymer 34, 1216 (1993).

${ }^{13} \mathrm{G}$. R. Hamed and M. Y. Huang, RubBer ChEM. TEChNOL. 71, 846 (1998).

${ }^{14}$ W. L. Hergenrother, J. Appl. Polym. Sci. 32, 3039 (1986); 3683 (1986).

${ }^{15}$ C. M. Roland, Rubber Chem. Technol. 62, 863 (1989).

${ }^{16}$ A. N. Gent in “Science and Technology of Rubber," $2^{\text {nd }}$ edition, J. E. Mark, B. Erman, and F. R. Eirich, Eds., Academic Press, New York, 1994, Ch. 10.

${ }^{17}$ G. J. Lake, Rubber Chem. Technol. 68, 435 (1995).

${ }^{18}$ A. N. Gent, P. B. Lindley, and A. G. Thomas, J. Appl. Polym. Sci. 8, 455 (1964).

${ }^{19}$ I. S. Choi and C. M. Roland, RubBer Chem. Technol. 69, 591 (1996).

${ }^{20}$ P. G. Santangelo and C. M. Roland in "Biopolymers from Renewable Resources," D.L. Kaplan, Ed., Springer-Verlag, Berlin, 1998, Ch. 14.

${ }^{21}$ I. S. Choi and C. M. Roland, RubBer Chem. TeChnol. 70, 202 (1997).

${ }^{22}$ G. J. Lake and P. B. Lindley, J. Appl. Polym. Sci. 8, 707 (1964).

${ }^{23}$ G. J. Lake and P. B. Lindley, J. Appl. Polym. Sci. 10, 343 (1966).

${ }^{24}$ C. M. Roland and J. W. Sobieski, Rubber Chem. TeChnol. 62, 683 (1989).

${ }^{25} \mathrm{P}$. H. Mott and C. M. Roland, Macromolecules 33, 4132 (2000). 